\title{
Two-Sided Test
}

National Cancer Institute

\section{Source}

National Cancer Institute. Two-Sided Test. NCI Thesaurus. Code C64267.

A statistical hypothesis test that controls directional errors if rejection of a hypothesis is the result of a directional decision. The two-sided test has less statistical power than the one-sided test. 